\title{
A naming convention for the Piano Key Weirs geometrical parameters
}

\author{
J. Pralong, J. Vermeulen, B. Blancher, F. Laugier \\ EDF - Hydro Engineering Center, France
}

S. Erpicum, O. Machiels, M. Pirotton

Hydrology, Applied Hydrodynamics and Hydraulic Constructions (HACH), University of Liege, Belgium

J-L. Boillat, M. Leite Ribeiro, A. Schleiss

Laboratory of Hydraulic Constructions (LCH), Ecole Polytechnique Fédérale de Lausanne, Switzerland

\begin{abstract}
Flood management is more than ever an issue for dam designers and engineering consulting firms in charge of rehabilitation works. Piano Key Weirs are a new cost-effective type of spillway designed to improve dams discharge capacity. These structures are particularly attractive: they can easily be built on existing structures and enable very high discharge capacities. Therefore, Piano Key Weirs are nowadays studied worldwide. Piano Key Weir description involves a lot of geometrical parameters (more than 30), which designations are not already universally defined. A naming convention is required to enhance exchanges and cooperation between the numerous developers. A naming convention has been developed at EDF-Hydro Engineering Center in cooperation with the Laboratory of Hydraulic Constructions (LCH), Ecole Polytechnique Fédérale de Lausanne and the Laboratory of Hydrology, Applied Hydrodynamics and Hydraulic Constructions (HACH), University of Liege. This paper describes the proposed naming convention and gives definitions and notations of the various geometrical parameters. This work represents a first attempt which should be updated with the contribution of stakeholders involved in this topic.
\end{abstract}

\section{INTRODUCTION}

In the frame of rehabilitation programs and new dams design, Piano Key Weirs represent a new advantageous alternative for spillways. PKW provide very high discharge capacity and can easily be built on the crest of existing dams. Moreover, they represent a cost-effective option for designers. This explains the worldwide interest for PKW development and implementation.

The scientific interest about PKW requires a unicity in terminology. Indeed, PKW geometry involves a lot of parameters making its configuration description uneasy.

A naming convention needs to be applied by the different stakeholders involved in PKW development. A workgroup gathering $E D F-H y d r o$ Engineering Center in cooperation with the Laboratory of Hydraulic Constructions (LCH), Ecole Polytechnique Fédérale de Lausanne and the Laboratory of Hydrology, Applied Hydrodynamics and Hydraulic Constructions (HACH), University of Liege developed a specific nomenclature. The naming convention aims to propose a uniform description to designers while keeping the number of parameters to a reasonable amount. The idea is to describe the general shape of PKW with a fixed number of geometrical inputs.

\section{PRINCIPLES OF THE NAMING CONVENTION}

The naming convention only cares about the structure of the PKW in itself. It does not consider any annex structures that could be required in some cases (bridge pier, anchorage, etc.). This 
nomenclature does not describe the lateral boundaries of the PKW neither as these parts are likely to differ from an implementation to another. However, it includes notations for physical parameters such as head or depths.

The structure of the PKW can be described as the gathering of different elements. The components considered in this nomenclature are the basic structure, the parapet walls and the noses (Fig. 1 left). The inlet keys, outlet keys and sidewalls compose the basic structure (Fig. 1 right) while noses and parapet walls are both optional in the PKW design. They are however considered in order to define as much configurations as possible.
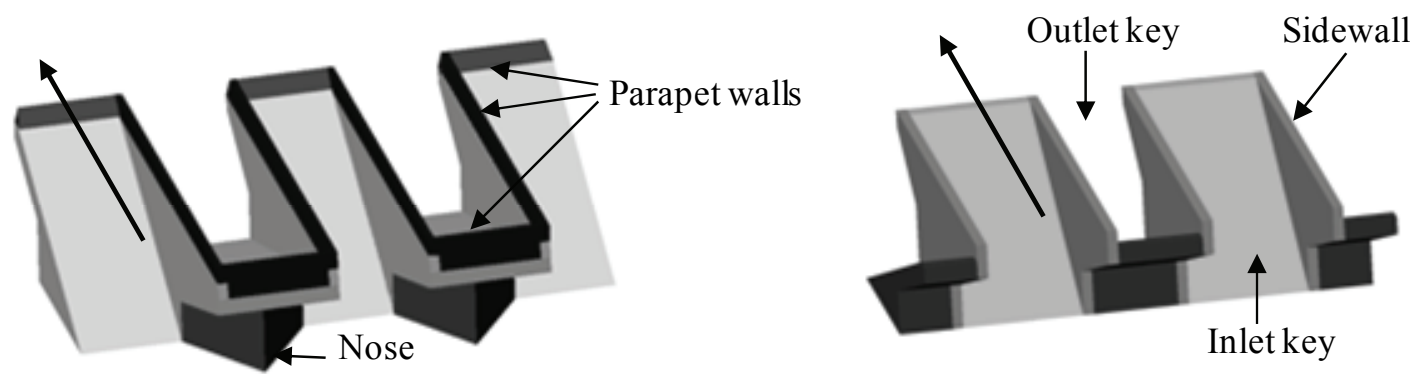

Figure 1: Components considered in the convention (left) and constitutive basic elements (right)

In order to keep the nomenclature as simple as possible, the number of geometrical parameters was limited to fundamental characteristics. The choice has been made to assign an index to the notations referring to the component or to the subcomponent (inlet key, outlet key, etc.) they are part of. Some of the parameters are overlapping but it has appeared that they all have advantages depending on circumstances. The number of parameters is thus not a minimum, but still remains reasonable.

The proposed description of the PKW structure relies on the assumption that PKW have a regular shape that can be divided into similar representative "PKW units" (Fig. 2 left). The unit represents the smallest extent of a complete structure and is composed of an entire inlet key with a sidewall and half an outlet key on both sides (Fig. 2 right).

This PKW unit is interesting as the whole structure can be reconstituted from it by juxtaposition. It is sufficient to describe most of the geometrical parameters of PKW. Only a few global dimensions need to be added to the unit description to fully define a PKW configuration.
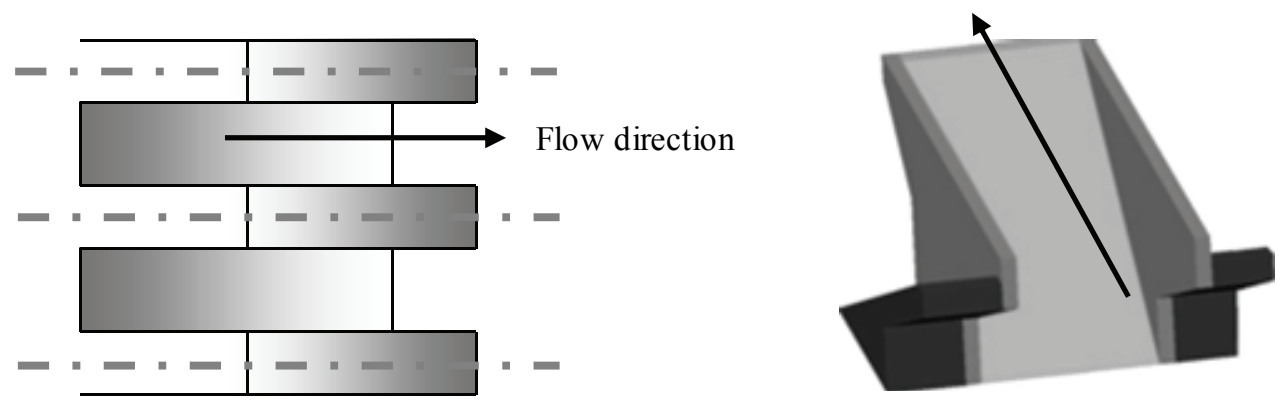

Figure 2: Plan view of the segmentation principle (left) and 3D-view of a PKW unit (right)

\section{FUNDAMENTAL PARAMETERS}

All the parameters described in this part are related to the global structure of the PKW. They are the most commonly used and reveal sufficient to provide an overall description of the weir. The parameters can be divided into categories depending on whether they represent lengths, widths, heights or thicknesses. In accordance with the nomenclature used for Labyrinth weirs (Falvey, 2003) and considering different previous publications about Labyrinth and PK-Weirs (US Dpt. of Interior 1987, Tullis 1995, Lempérière \& Ouamane 2003, Leite Ribeiro et al. 2009) the following rules have been applied: $B$ is used for lengths; $W$ for widths; $P$ for heights; $L$ for the developed lengths; $S$ for slopes and $T$ for thicknesses. 
Indexes are applied to define the location of the geometrical parameter: for instance, index $i$ refers to inlet key; index $o$ stands for outlet key and index $b$ refers to parameters linked to the base of the structure.

Based on these rules, 23 parameters have been defined to describe the PKW global structure general shape (Fig. 3 to Fig. 6 and Tab. 1). Parapet walls are taken into account as they influence the height of the weir, but are not described in this part.

The last five parameters of Table 1 are not required for the PKW description as they can be deduced from the previous ones. However, they are interesting for comparisons between configurations as they give a first idea of the dimensions and geometric aspect of the weir.

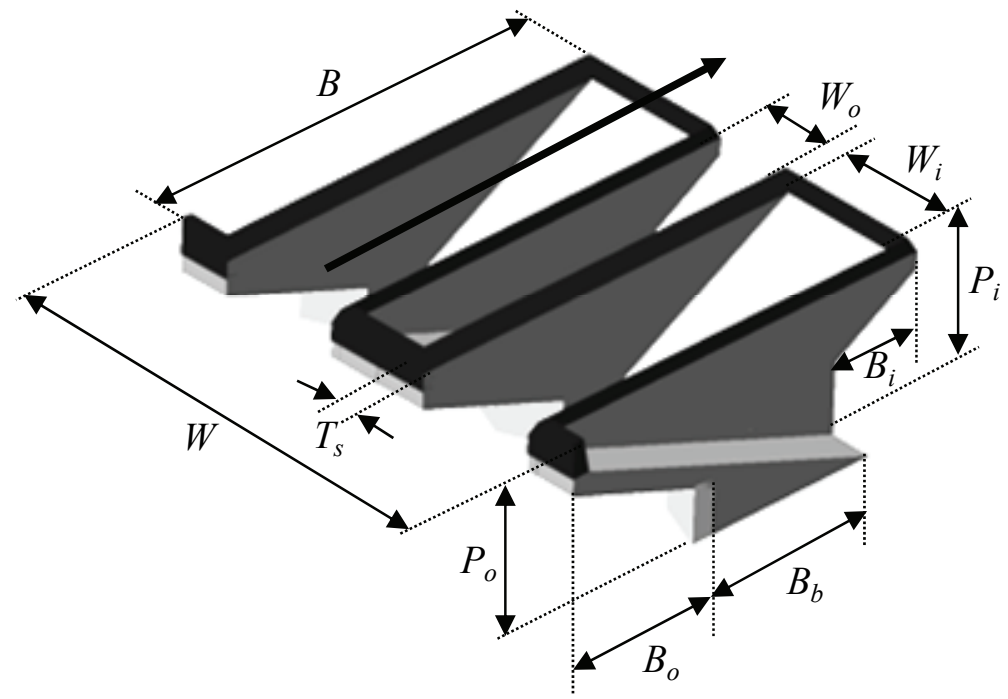

Figure 3: Fundamental parameters on an entire PKW - 3D-view
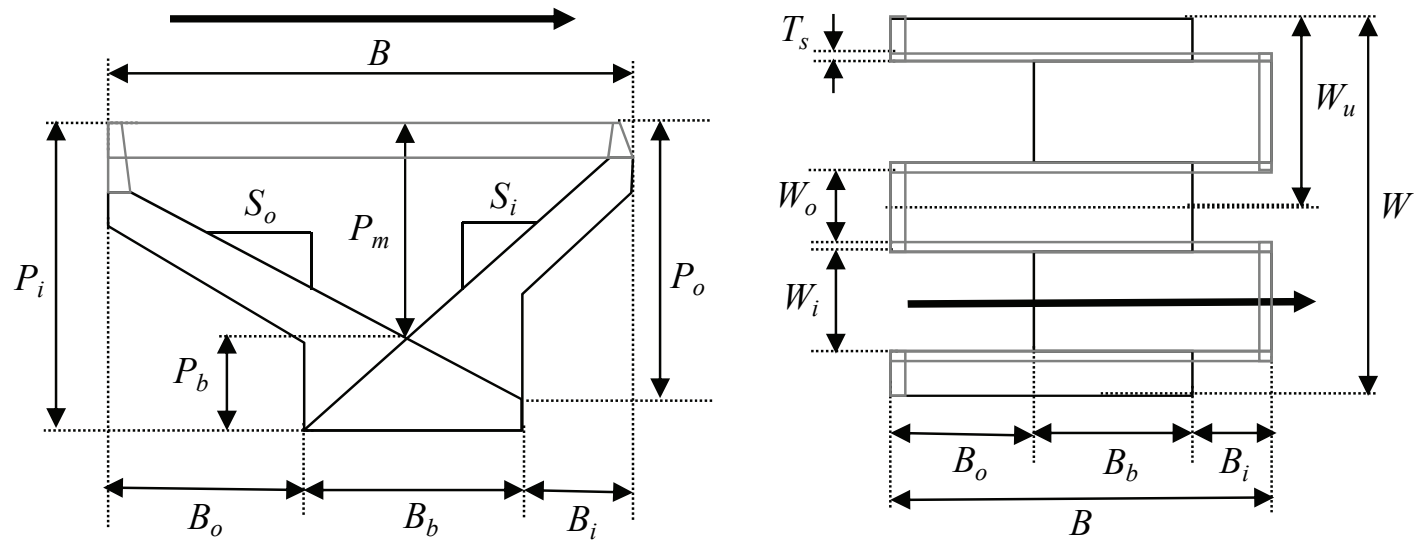

Figure 4: Fundamental parameters on an entire PKW - plan view (left) and cross section (right)

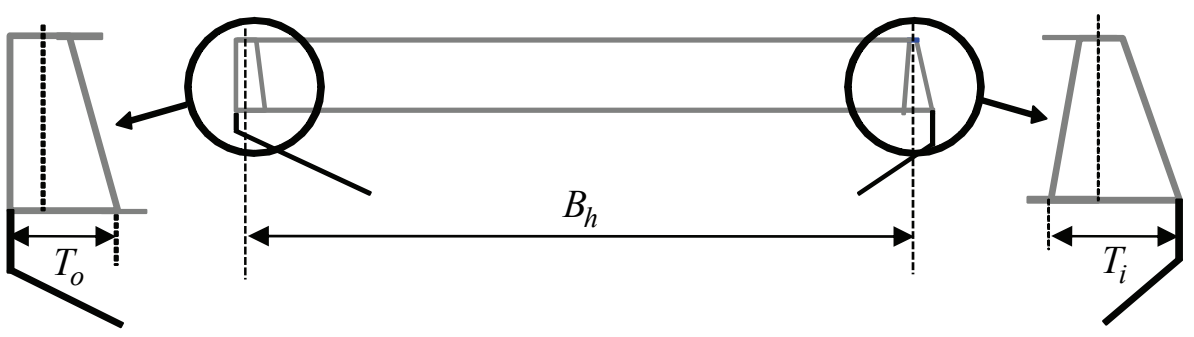

Figure 5: Detail cross sections of the PKW crests 

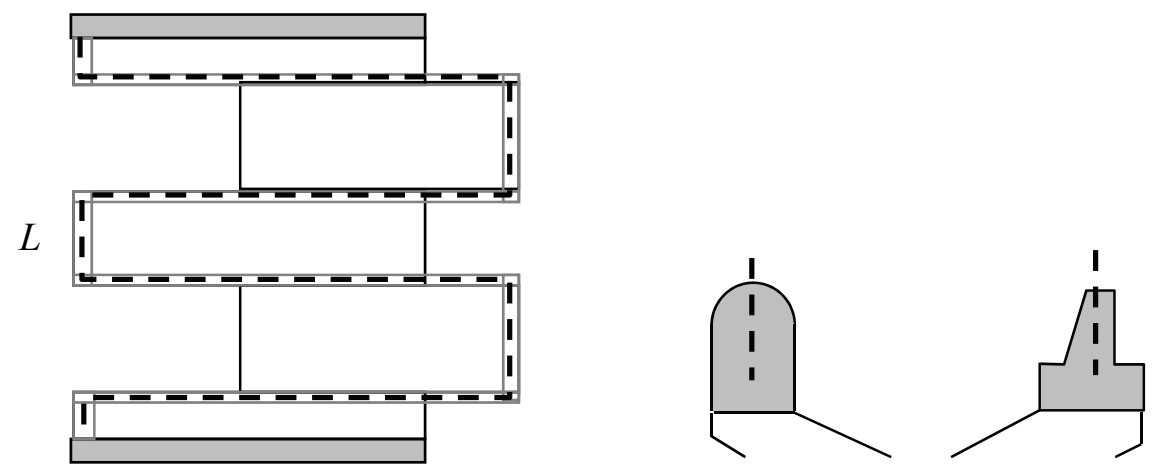

Figure 6: Plan view of parameter $L$ over an entire PKW (left) and typical crest cross sections (right)

Table 1: Nomenclature of the fundamental parameters of PKW geometry

\begin{tabular}{|c|c|}
\hline Parameter symbol & Meaning \\
\hline$B$ & Upstream-downstream length of the PKW $B=B_{b}+B_{i}+B_{o}$ \\
\hline$B_{o}$ & Upstream (outlet key) overhang crest length \\
\hline$B_{i}$ & Downstream (inlet key) overhang crest length \\
\hline$B_{b}$ & Base length \\
\hline$B_{h}$ & $\begin{array}{l}\text { Sidewall overflowing crest length measured from the outlet key } \\
\text { crest axis to the inlet key crest axis (Fig. 5) }\end{array}$ \\
\hline$P_{i}$ & $\begin{array}{l}\text { Height of the inlet entrance measured from the PKW crest (includ- } \\
\text { ing possible parapet walls) }\end{array}$ \\
\hline$P_{o}$ & $\begin{array}{l}\text { Height of the outlet entrance measured from the PKW crest (includ- } \\
\text { ing possible parapet walls) }\end{array}$ \\
\hline$P_{b}$ & Height of the apron level at inlet key and outlet key intersection \\
\hline$P_{m}$ & Difference between $P_{i}$ and $P_{b}$ \\
\hline$S_{i}$ & Slope of the inlet key apron (length over height) \\
\hline$S_{o}$ & Slope of the outlet key apron (length over height) \\
\hline$W$ & Total width of the PKW \\
\hline$W_{u}$ & Width of a PKW unit \\
\hline$W_{i}$ & Inlet key width (sidewall to sidewall) \\
\hline$W_{o}$ & Outlet key width (sidewall to sidewall) \\
\hline$T_{s}$ & Sidewall thickness \\
\hline$T_{i}$ & $\begin{array}{l}\text { Horizontal crest thickness at inlet key extremity (measured at the } \\
\text { basis of possible parapet walls) }\end{array}$ \\
\hline$T_{0}$ & $\begin{array}{l}\text { Horizontal crest thickness at outlet key extremity (measured at the } \\
\text { basis of possible parapet walls) }\end{array}$ \\
\hline$L$ & Total developed length along the overflowing crest axis \\
\hline$L_{u}$ & $\begin{array}{l}\text { Developed length of the PKW unit along the overflowing crest axis } \\
L_{u}=W_{i}+W_{o}+2 B_{h}+2 T_{S}\end{array}$ \\
\hline$N_{u}$ & Number of PKW units constituting the structure \\
\hline$n$ & Developed length ratio of the PKW : $\quad n=\frac{L}{W}$ \\
\hline$n_{u}$ & Developed length ratio of a PKW unit : $n_{u}=\frac{L_{u}}{W_{u}}$ \\
\hline
\end{tabular}


Some physical parameters also need to be added to this naming convention. The proposed selection has been made to limit their number to a minimum (Fig. 7 and Tab. 2).

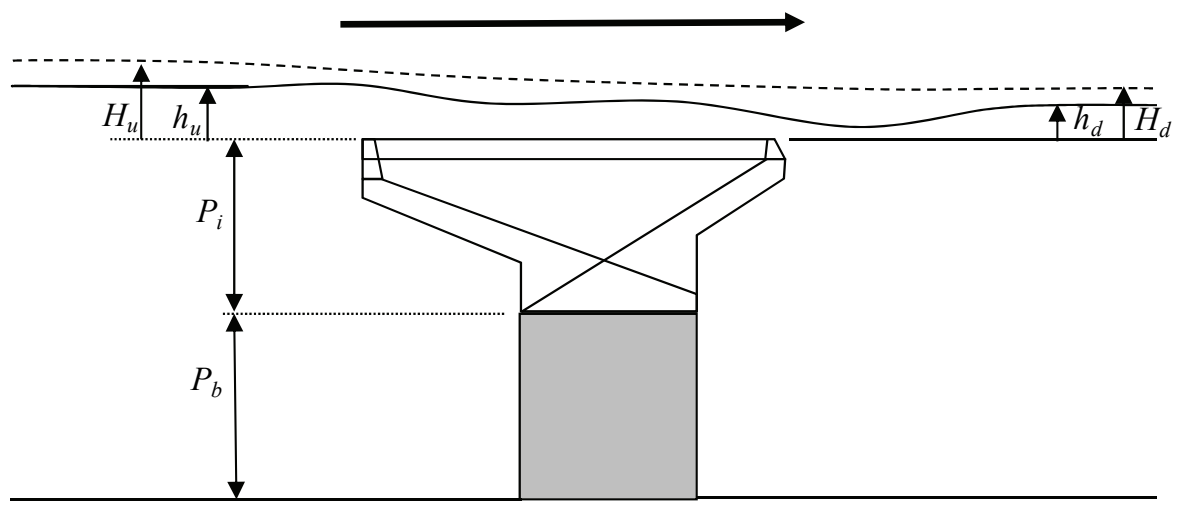

Figure 7: Cross section of the physical parameters nomenclature

Table 2: Physical parameters nomenclature

\begin{tabular}{l|l}
\hline Parameter symbol & Meaning \\
\hline$P_{d}$ & Dam height \\
\hline$H_{u}$ & Total head over crest upstream from weir \\
\hline$h_{u}$ & Upstream flow depth over crest \\
\hline$H_{d}$ & Total head over crest downstream from weir (can be negative) \\
\hline$H_{d}$ & Downstream flow depth over crest (can be negative) \\
\hline$Q$ & Flow discharge \\
\hline$q_{s W}$ & Specific discharge referred to total width of the PKW $q_{s W}=\frac{Q}{W}$ \\
\hline$C_{d W}$ & $\begin{array}{l}\text { Discharge coefficient related to PKW total width as } \\
\text { Q }\end{array}$ \\
\hline$q_{s L}$ & $\begin{array}{l}\text { Specific discharge referred to developed length of the PKW } \\
q_{s L}=\frac{Q}{L}\end{array}$ \\
\hline$C_{d L}$ & $\begin{array}{l}\text { Discharge } \quad \text { coefficient related to PKW developed length as } \\
Q=C_{d L} \cdot L \cdot \sqrt{2 g} \cdot H^{3 / 2}\end{array}$ \\
\hline
\end{tabular}

\section{OPTIONAL DETAIL PARAMETERS}

The fundamental parameters above enable to describe the global configuration of the PKW, but are not sufficient to define nor the geometry of optional features nor the structure of the overhangs. Additional parameters are then necessary to complete a full description of the structure. They are presented hereafter, based on the current knowledge about PKW optimization.

\subsection{Overhangs structural description}

There is a need for engineers to be able to assess concrete volumes involved in a PKW structure. This requires a description of overhangs thicknesses. Moreover overhangs mass balance is crucial for PKW stability. The information provided by the description should enable a first assessment of these two items. The proposed parameters concern the thicknesses of the overhangs and the shape at their extremities (Fig. 8, Fig. 9 and Tab. 3). Index $b$ still refers to the base of the PKW and index $c$ stands for the crest of the keys. 

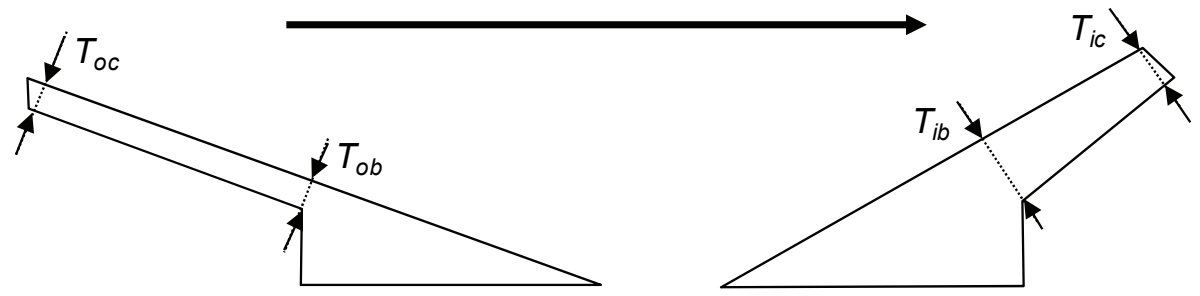

Figure 8: Overhangs base and extremities thicknesses - upstream (left) and downstream (right)
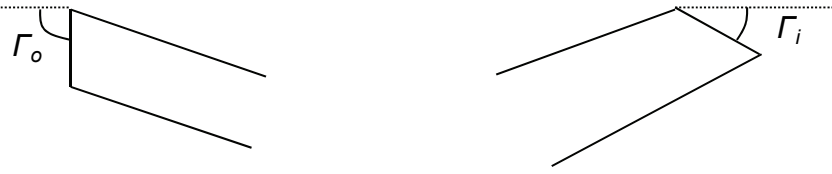

Figure 9: Overhangs extremity batter angle - upstream (left) and downstream (right) cross sections

Table 3: Nomenclature of overhangs thicknesses and batter angle parameters

\begin{tabular}{c|l}
\hline Parameter symbol & Meaning \\
\hline$T_{o b}$ & Upstream overhang thickness at upstream base extremity \\
\hline$T_{o c}$ & Upstream overhang thickness at upstream extremity \\
\hline$T_{i b}$ & Downstream overhang thickness at downstream base extremity \\
\hline$T_{i c}$ & Downstream overhang thickness at downstream extremity \\
\hline$\Gamma_{o}$ & Batter angle at upstream (outlet key) overhang extremity \\
\hline$\Gamma_{i}$ & Batter angle at downstream (inlet key) overhang extremity \\
\hline
\end{tabular}

\subsection{Parapet walls}

Parapet walls have been developed while optimizing the hydraulic efficiency of PKW. They consist of vertical extensions that can be placed over the crest of the PKW. Three components of parapet wall can be distinguished: the inlet key one, the outlet key one, and the sidewall one. The two first enable to modify the longitudinal cross-sections of the keys while the latter especially aims in modifying the crest profile of the sidewall. The three components are independent, and do not need to have the same height. In this nomenclature, index $p$ refers to parapet walls.

The naming convention describes each of the three features following the same parameterization. Then, index $i$ refers to the downstream parapet wall of inlet key, index $o$ to the upstream parapet wall of outlet key, and index $s$ to sidewall parapet wall. Hence, for $x \in[i, o, s]$ (Fig. 10 and Tab. 4).
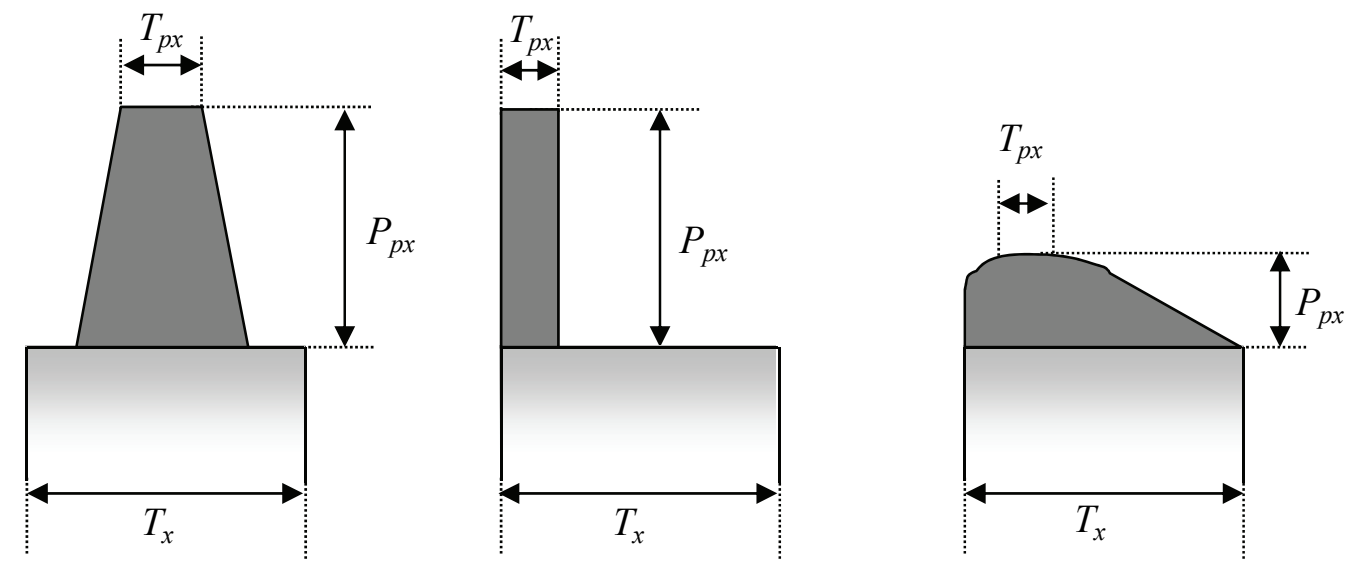

Figure 10: Parapet walls parameters in cross section 
As shown on Figure 10, there is an infinite number of possible shapes for parapet walls. The two proposed parameters are obviously not enough to fully describe the geometry of the parapet walls. This nomenclature cannot take into account such a variety of shapes. The naming convention gives the global dimensions of the parapet walls and recommends to the authors to provide a detailed scheme to define their geometries.

Table 4: Nomenclature of parapet walls parameters

\begin{tabular}{c|l}
\hline Parameter symbol & Meaning \\
\hline$P_{p x}$ & Height of the parapet wall on x-component \\
\hline$T_{p x}$ & Crest thickness of the parapet wall on x-component \\
\hline
\end{tabular}

\subsection{Noses and side wall angle}

Noses are features placed under the upstream overhangs in order to increase the discharge capacity of PKW by improving the flow pattern at inlet keys entrance. Their shape can vary from triangular profile to rounded one. In this naming convention, their description only requires one parameter (Fig. 11 left and Tab. 5), but the nose shape needs to be mentioned. In the case when other parameters would be required to define the nose shape, they would be characterised with index $n$.

Labyrinth design is familiar with the notion of sidewall angle $\alpha$. This parameter can be transposed to PKW structures. Indeed, it is likely to improve the discharge capacities, especially for high upstream heads. The notation used by Falvey (2003) has been adopted for PKW (Fig. 11 right and Tab. 5).
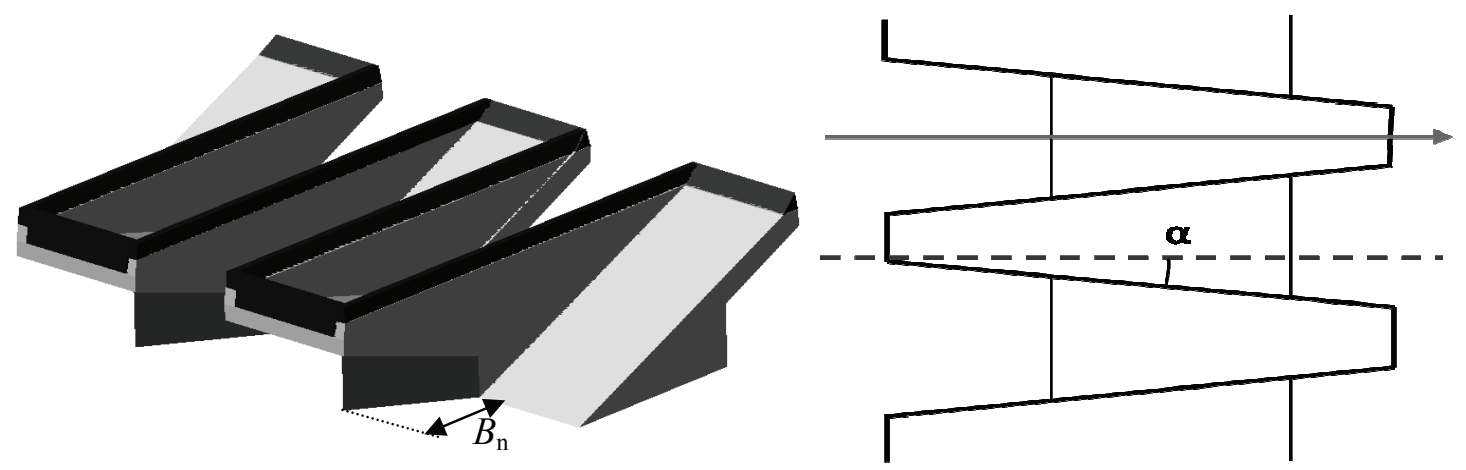

Figure 11: Nose parameter -3D-view (left) and sidewall angle - plan view (right)

Table 5: Nomenclature of nose parameter and sidewall angle

\begin{tabular}{l|l}
\hline Parameter symbol & Meaning \\
\hline$B_{n}$ & Length of the nose \\
\hline \hline$\alpha$ & Sidewall angle \\
\hline
\end{tabular}

\section{REFERENCE LAYOUTS}

The global cross section layout enables to distinguish several categories of PKW.. A basic classification has been proposed by Lempérière a few years ago with respect to the arrangement of the upstream and downstream overhangs (Fig. 12):

Type A - with symmetrical upstream and downstream overhangs

Type B - with only upstream overhang

Type C - with only downstream overhang 

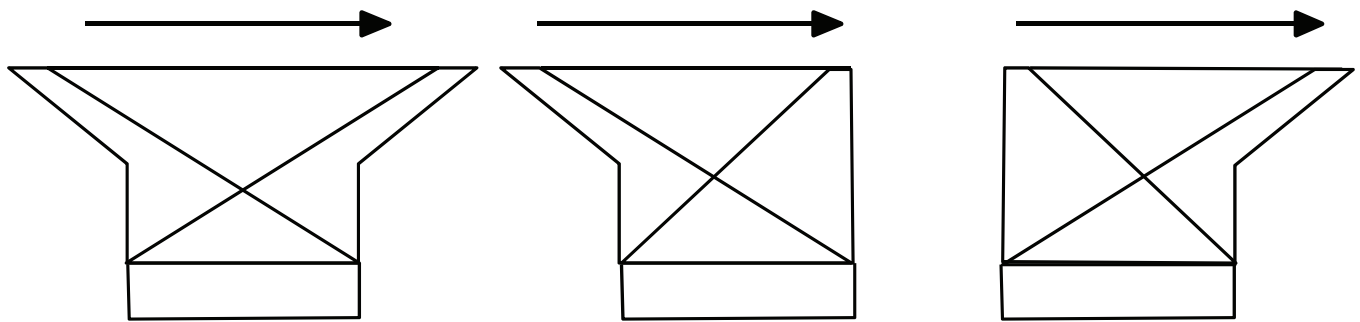

Figure 12. Typical shapes of PKW: Type A (left); Type B (middle); Type C (right)

\section{CONCLUSIONS}

The developed naming convention enables to describe globally a PKW using 22 parameters. Additional parameters allow engineers and developers to assess concrete volume and mass balance and to integrate optional features to their design.

Recent PKW optimization investigations have focused on noses and parapet walls, and this is the reason why specific parameters are proposed for these optional features. Due to the variety of designs, this nomenclature recommends to join schemes to the descriptions of special features or new design options.

Future developments will probably introduce other particularities and require completing the parametric description. The naming convention will have to evolve with PKW development, but already accounts for a very large number of PKW configurations.

\section{AKNOWLEDGEMENTS}

Special thanks are extended to F. Lempérière, M. Ho Ta Kanh, G. Degoutte and J. P. Tullis for their helpful contributions to the redaction of this naming convention.

\section{REFERENCES}

Falvey, T. H.. (2003). Hydraulic design of labyrinth weirs. ASCE Press.

Lempérière, F., Ouamane, A.. (2003). The PK Weir : a new cost-effective solution for spillways. Hydropower \& Dams, Issue five.

Tullis, J. P.. (1995). Design of Labyrinth Spillways. Journal of Hydraulic Engineering.

United States Department of Interior, (1987). Design of Small Dams. A water resources technical publication, Third edition.

Leite Ribeiro, M., Bieri, M., Boillat, J-L., Schleiss, A., Delorme, F. and Laugier, F. (2009). "Hydraulic capacity improvement of existing spillways - Design of piano key weirs". 23rd Congress of Large Dams. Question 90, Response 43. 25-29 May 2009. Brasilia, Brazil. 